Istituto di Genetica dei Tumori "Cassa di Risparmio PP. LL." del Centro di Studi di Genetica Umana dell'Università di Milano

Direttore: Prof. Luisa Gianferrari

\title{
Ricerche sulla familiarità del carcinoma ovarico
}

\author{
I. Osservazioni sui casi primari e rilievi sull'età di manifestazione \\ dei carcinomi ovarici e sulla fertilità delle pazienti affette da cancro dell'ovaio
}

\section{A. Soini e A. Serra}

Non sono mancate in passato relazioni di casi nè ricerche genealogiche intese a contribuire alla soluzione del problema della familiarità ed ereditarietà delle neoplasie ovariche nella specie umana. Limitandoci alle forme maligne, ricordiamo prima di tutto la casistica gemellare; classificata da Gedda (I95I) fino al I949: 2 casi di carcinoma ovarico concordante in due coppie di gemelle $\mathrm{MZ}$, osservati da Kimbrough nel 1928 e da McFarland e Meade nel I932; il caso di carcinoma ovarico discordante, in cui una sola delle gemelle $M Z$ fu colpita, riferito da Versluys nel I934; una coppia DZ concordante per fibrosarcoma ovarico ed una coppia DZ bisesso relativamente concordante ( $\sigma^{*}$ con cancro dello stomaco e $q$ con fibrosarcoma ovarico), segnalate da Waaler nel i93 I; le due coppie DZ bifemminili relativamente concordanti descritte da Kranz nel i932, nelle quali una delle cogemelle era affetta da cancro ovarico e le altre due erano state rispettivamente colpite da cancro uterino e da neoplasie multiple; ed infine la coppia DZ bifemminile relativamente concordante (una con cancro ovarico e l'altra con cancro uterino) della serie gemellare di Habs, resa nota nel I939. A questa casistica va aggiunta una coppia $M Z$ discordante per cancro ovarico appartenente alla serie studiata da Von Verschuer e Kober (I956).

Tra i casi familiari uno soltanto è stato finora oggetto di pubblicazione, probabilmente per la sua straordinarietà : è il caso di una madre e 5 figlie su 7 , tutte affette da cancro ovarico, presentato - con tentativi di interpretazione sul tipo della trasmissione ereditaria - da Liber (1950).

$\mathrm{Va}$ accennata per ultima l'ampia indagine condotta con metodo genealogico da Abruzzese (1934) sull'ereditarietà dei tumori femminili. Per quanto riguarda il cancro ovarico, pur ammettendo che «sarebbe necessario un materiale molto più numeroso » (p. 24) per raggiungere risultati più attendibili, egli afferma che è la neoplasia che presenta « il più alto coefficiente di eredità specificatamente cancerosa " (p. 97): infatti il suo «Indice ereditario neoplastico », cioè la percentuale delle pazienti di cancro ovarico nella cui parentela (nonni, genitori, zii, fratelli) ha trovato almeno un altro caso di tumore maligno, raggiunge il $47,6 \%$. 
Questa sommaria sintesi della letteratura lascia per se stessa trasparire l'incertezza delle conclusioni raggiunte e di ogni soluzione prospettata: la scarsità del materiale studiato e l'incompletezza dell'analisi, dovuta principalmente alla difficoltà della raccolta dei dati ad essa necessari, sono troppo evidenti. Non appare strano quindi che Novak dell'Ovarian Tumor Registry dichiarasse nel 1944, in risposta a domande di informazioni da parte del Liber (I950), di non possedere casi che illustrassero alcuna tendenza familiare e che Selye nel 1946 scrivesse che « vi sono poche prove per sostenere l'esistenza di un importante fattore ereditario nello sviluppó del carcinoma ovarico».

Le ricerche da noi iniziate ed eseguite con metodo genealogico-statistico vorrebbero precisamente rappresentare un contributo all'accertamento dell'esistenza o meno di una significativa concentrazione di forme neoplastiche omotope ed eterotope nelle famiglie di blastomatose ovariche: primo dato essenziale in uno studio organico sull'eredità della forma neoplastica considerata.

Si sono presentate due particolari difficoltà. Prima, la notevole difformità della terminologia diagnostica, causata soprattutto dalla complessità e dalla molteplicità delle classificazioni delle neoplasie ovariche (Schiller, I940; Malcovati, 1948; Nègre, I 948; Barzilai, I949; Novak, I953; Ferraris e col., I955): complessità e molteplicità che trovano una spiegazione nelle particolari caratteristiche anatomo-istologiche dell'ovaio e nei diversi criteri di classificazione adottati: embriogenetico, morfologico, clinico, anatomochirurgico. Seconda, l'incertezza di varie diagnosi, a causa della non sempre facile distinzione, anche a reperto istologico eseguito, tra forme benigne e maligne per il graduale evolversi delle une nelle altre e tra forme metastatiche e primitive.

Perciò richiedendo l'indagine genealogica necessaria al raggiungimento del fine propostoci la omogeneità del gruppo delle pazienti rispetto alla forma in istudio, cioè che tutte siano state affette da neoplasie ovariche maligne non metastatiche e di una certa affinità istologica, è sembrato utile esaminare un poco particolareggiatamente - col sussidio della tecnica meccanografica - il nostro materiale. Si è cosi definito il gruppo dei «casi primari» e se ne è completata poi la caratterizzazione con lo studio di due elementi di notevole valore dal punto di vista genetico: l'età di manifestazione della malattia e la fertilità delle donne colpite.

\section{I casi primari}

I casi di tumori ovarici maligni da noi raccolti mediante lo spoglio sistematico e l'esame delle cartelle cliniche ${ }^{1}$ sono in totale 296 . Di questi: i 73 erano stati ricoverati presso la Clinica Ostetrico-Ginecologica dell'Università di Milano nel periodo

1 Ringraziamo vivamente il Prof. Mario Massazza Direttore della Clinica Ostetrico-Ginecologica dell’Università di Milano, il Prof. Eugenio Maurizio Direttore della Clinica Ostetrico-Ginecologica dell'Università di Genova, il Prof. Piero Malcovati Direttore dell'Istituto Provinciale di Maternità di Milano ed il Prof. Giuseppe Vecchietti Primario della Divisione Ostetrico-Ginecologica dell'Ospedale Maggiore di Vercelli per aver favorito e facilitato la ricerca mettendoci a disposizione le cartelle cliniche ed i registri delle diagnosi istologiche. 
Soini A., Serra A.: Ricerche sulla familiarità del carcinoma ovarico

Tabella I - Frequenza dei tumori ovarici maligni secondo il tipo morfologico

\begin{tabular}{|c|c|c|c|c|c|c|}
\hline \multirow[b]{2}{*}{ Tipo morfologico } & \multicolumn{4}{|c|}{ Numero dei casi } & \multirow{2}{*}{\multicolumn{2}{|c|}{ Totale }} \\
\hline & \multicolumn{2}{|c|}{$\begin{array}{l}\text { con diagnosi } \\
\text { istologica }\end{array}$} & \multicolumn{2}{|c|}{$\begin{array}{l}\text { senza diagnosi } \\
\text { istologica }\end{array}$} & & \\
\hline & $\mathrm{n}$ & $\%$ & $\mathrm{n}$ & $\%$ & $\mathbf{n}$ & $\%$ \\
\hline Carcinomi solidi primitivi & $5^{\circ}$ & 41,32 & 24 & $3 \mathrm{I}, \mathrm{I} 7$ & 74 & 37,37 \\
\hline Cistocarcinomi & 60 & 49,59 & $4^{8}$ & 62,34 & 108 & 54,54 \\
\hline Carcinomi metastatici & I I & 9,09 & 5 & 6,49 & 16 & 8,09 \\
\hline Totali & I 2 I & 100,00 & 77 & 100,00 & 198 & 100,00 \\
\hline Sarcomi & 4 & - & 一 & 一 & 4 & - \\
\hline Teratomi & $\mathbf{I}$ & - & 一 & - & $\mathbf{I}$ & - \\
\hline Tumori maligni non classificati & 一 & - & 93 & - & 93 & - \\
\hline Totali & I 26 & & $17^{\circ}$ & & 296 & \\
\hline
\end{tabular}

I946-I955; 66 presso l'Istituto Provinciale di Maternità di Milano nel periodo I946I 955; 28 presso la Clinica Ostetrico-Ginecologica dell'Università di Genova nel periodo I950-I955; 29 presso la divisione Ostetrico-Ginecologica dell'Ospedale Maggiore di Vercelli nel periodo i950-1955.

Il tipo istologico della neoplasia si è potuto stabilire con certezza soltanto per I 26 casi, al cui riguardo si disponeva di reperto e diagnosi istologica. Per questo gruppo, come appare dalla tabella I, buona risulta la corrispondenza con le casistiche del Novak (1953) e dello Schmid (1954) circa la frequenza dei cistocarcinomi, del $58 \%$ nel primo e del $50 \%$ nel secondo. Sembra invece esistere una notevole diversità nelle frequenze dei carcinomi solidi primitivi e metastatici: secondo Schmid (I954) gli uni costituirebbero il $27 \%$ gli altri il $23 \%$ di tutti i carcinomi. La diversità trova una plausibile spiegazione nell'influsso esercitato sulla composizione del campione, generalmente non molto grande, da parte di molti fattori tra cui - nel caso particolare - il più facile ricovero dei metastatici dove si erano subiti precedenti interventi piuttosto che in reparti di specialità ginecologica.

Per gli altri I 70 casi, per i quali o non era stata effettuata la diagnosi istologica o non è stato possibile rintracciare nè la diagnosi istologica nè il reperto istologico, si è accettata come valida la diagnosi intraoperatoria o quella clinica. Se ne distinguono (tabella I) due gruppi. Il primo è costituito dai casi morfologicamente classificati. La differente proporzione dei carcinomi solidi, cistocarcinomi e carcinomi metastatici qui riscontrata, rispetto a quella del gruppo precedente, sembra mantenersi entro i limiti di una variazione casuale, non risultando dalla prova dell'omogeneità per campioni paralleli (Rao, I952) una differenza significativa tra le due distribuzioni (tabella 2). Il secondo gruppo è formato invece da 93 casi morfologicamente non classificati. Anche-di questi ultimi è sembrato lecito tener conto nella successiva indagine genealogica. Anzitutto non c'è dubbio sulla loro malignità: ne 
Acta Geneticae Medicae et Gemellologiae

Tabella 2 - Prova dell'omogeneità dei due campioni di carcinomi morfologicamente classificati

\begin{tabular}{|c|c|c|c|c|}
\hline & $\begin{array}{l}\text { Carcinomi } \\
\text { solidi }\end{array}$ & Cistocarcinomi & $\begin{array}{l}\text { Carcinomi } \\
\text { metastatici }\end{array}$ & Totale \\
\hline Senza diagnosi istologica $\left(n_{1}\right)$ & 24 & 48 & 5 & 77 \\
\hline Con diagnosi istologica $\quad\left(n^{\prime}{ }_{1}^{\prime}\right)$ & $5^{\circ}$ & 60 & I I & 121 \\
\hline Totali $\left(\underline{n_{1}+n_{1}^{\prime}}\right)$ & 74 & 108 & 16 & 198 \\
\hline$p_{i} \quad\left(\frac{n_{1}}{n_{i}+n^{\prime}}\right)$ & 0,4800 & 0,8000 & 0,4545 & 0,6364 \\
\hline
\end{tabular}

depongono a favore in modo speciale le elevate frequenze di bilateralità, metastasi e ascite (tabella 3) corrispondenti a quelle dei due gruppi di carcinomi istologicamente e clinicamente definiti ed in sufficiente accordo con $\mathrm{i}$ dati di altri AA. sui carcinomi ovarici in genere. Così il Lynch (1936) su I Io casi e Montgomery (1948) su 107 ne trovarono rispettivamente il $60 \%$ ed il $70 \%$ con bilateralità; mentre Allan e coll. (I 948 ) su 265 casi ne osservarono $32, \mathrm{I} \%$ con bilateralità, il $52 \%$ con metastasi ed il $30,9 \%$ con ascite.

Inoltre 48 delle 93 pazienti sono state sottoposte ad intervento operatorio: 20 esplorativo, 3 con ovariectomia monolaterale, 7 con ovariectomia bilaterale, I 7 con ovariectomia ed isterectomia subtotale, I con ovariectomia ed isterectomia totale. Riguardo alle altre 45:29 non hanno subito intervento, ma di queste I4 sono state irradiate; di i 6 non risultano ulteriori notizie dalle cartelle cliniche.

Circa l'affinità istologica tra $\mathrm{i}$ casi di questo gruppo e degli altri due non è possibile sollevare serie obiezioni. E noto che la frequenza di altre forme tumorali maligne dell'ovaio (sarcomi, teratomi, disgerminomi, ecc.) è minima rispetto ai carcinomi solidi e cistocarcinomi: è quindi ragionevolmente supponibile che le due ultime forme prevalgano in misura molto notevole tra i 93 casi di cui il gruppo è costituito.

Tabella 3 - Frequenza di alcune caratteristiche patologiche riscontrate nei tre sottogruppi delle 275 pazienti affette da tumore ovarico maligno

\begin{tabular}{|c|c|c|c|c|c|c|c|}
\hline \multirow{2}{*}{ Tipo morfologico } & \multirow{2}{*}{$\begin{array}{l}\text { N. di } \\
\text { casi }\end{array}$} & \multicolumn{6}{|c|}{ Numero dei casi con } \\
\hline & & \multicolumn{2}{|c|}{ bilateralità } & \multicolumn{2}{|c|}{ metastasi } & \multicolumn{2}{|c|}{ ascite } \\
\hline & & $\mathbf{n}$ & $\%$ & $\mathrm{n}$ & $\%$ & $\mathrm{n}$ & $\%$ \\
\hline Carcinomi solidi primitivi & 74 & $4 \mathrm{I}$ & $55,4 \mathbf{I}$ & $4^{8}$ & 64,86 & 24 & $3^{2,43}$ \\
\hline Cistocarcinomi & 108 & 55 & $5^{0,93}$ & 63 & $5^{8,33}$ & $3^{8}$ & 35,19 \\
\hline T. maligni non classificati & 93 & 39 & 41,94 & $5^{8}$ & 62,37 & 39 & $4 I, 94$ \\
\hline & 275 & I 35 & 49 , I & $\mathbf{I} 69$ & 61,45 & IOI & $3^{6,73}$ \\
\hline
\end{tabular}


In conclusione, questo breve esame del nostro materiale ci consente di ritenere 275 casi, sui 296 raccolti, come un gruppo sufficientemente omogeneo per lo scopo della nostra indagine e suddivisibile in tre sottogruppi:

\section{Carcinomi solidi primitivi 74 \\ II. Cistocarcinomi 108 \\ III. Tumori maligni non classificati 93}

Dalla successiva indagine genealogico-statistica sono stati quindi esclusi i i 6 casi di carcinomi metastatici, i 4 casi di sarcoma e l'unico caso di teratoma incontrati nella raccolta del materiale.

\section{Età di manifestazione dei carcinomi ovarici}

Un elemento di interesse clinico e genetico nello stesso tempo è l'età di manifestazione della forma neoplastica.

Le stime statistiche, riportate nella tabella 4, dove è data anche la distribuzione delle pazienti per età e per gruppo di appartenenza, indicano come età media di manifestazione, per il cancro ovarico in genere, $5^{\mathrm{I}, \mathrm{I}} 5 \pm 0,64$ anni; valore che si accorda, come si attenderebbe, con quello trovato per $\mathrm{i}$ tumori maligni non classificati $\left(5 \mathrm{I}, 5^{6}\right.$

Tabella 4 - Distribuzione delle pazienti per età alla diagnosi

\begin{tabular}{|c|c|c|c|c|c|c|c|c|}
\hline \multirow{3}{*}{$\begin{array}{l}\text { Classi } \\
\text { di età }\end{array}$} & \multicolumn{6}{|c|}{ Pazienti affetta da } & \multirow{2}{*}{\multicolumn{2}{|c|}{ Totale }} \\
\hline & \multicolumn{2}{|c|}{ Carcinoma solido } & \multicolumn{2}{|c|}{ Cistocarcinoma } & \multicolumn{2}{|c|}{$\begin{array}{c}\text { T. maligno } \\
\text { non classificato }\end{array}$} & & \\
\hline & $\mathrm{n}$ & $\Sigma \mathrm{f} \%$ & $\mathrm{n}$ & $\Sigma \mathrm{f} \%$ & $\mathbf{n}$ & $\Sigma \mathrm{f} \%$ & $\mathbf{n}$ & $\Sigma \mathrm{f} \%$ \\
\hline $10-15$ & - & - & 一 & 一 & $\mathbf{I}$ & I, I I & $I$ & 0,37 \\
\hline $15-20$ & 2 & 2,70 & - & - & - & - & 2 & $I, 10$ \\
\hline $20-25$ & 2 & $5,4^{I}$ & 一 & 一 & 一 & - & 2 & $\mathrm{I}, 84$ \\
\hline $25-30$ & I & 6,76 & - & - & I & 2,22 & 2 & 2,57 \\
\hline $3^{0-35}$ & 5 & $13,5 \mathrm{I}$ & 3 & 2,78 & 2 & 4,44 & 10 & 6,25 \\
\hline $35-40$ & 2 & 16,22 & 5 & 7,40 & 6 & I I , I I & 13 & I I , O3 \\
\hline $40-45$ & 9 & $28,3^{8}$ & 17 & 23,15 & IO & 22,22 & $3^{6}$ & 24,26 \\
\hline $45-5^{\circ}$ & I6 & $5^{0,00}$ & 22 & $43,5^{I}$ & 25 & $5^{0,00}$ & 63 & 47,43 \\
\hline $5^{0-55}$ & I 4 & 68,92 & I 8 & 60,18 & 13 & 64,44 & 45 & 63,97 \\
\hline $55^{-60}$ & I 3 & $86,4^{8}$ & 20 & 78,70 & Io & 75,55 & 43 & 79,78 \\
\hline $60-65$ & 6 & 94,59 & 14 & $9^{1,57}$ & I 5 & $9^{2,22}$ & 35 & $9^{2,}, 65$ \\
\hline $65-70$ & 3 & 98,64 & 5 & 96,96 & 2 & 94,44 & Io & 96,32 \\
\hline $70-75$ & I & I00,00 & 3 & 99,07 & 4 & $9^{8,88}$ & 8 & 99,26 \\
\hline $75^{-80}$ & - & - & $\mathrm{I}$ & 100,00 & $\mathbf{I}$ & 100,00 & 2 & 100,00 \\
\hline Totali & 74 & 100,00 & 108 & 100,00 & 90 & 100,00 & 272 & 100,00 \\
\hline Medie & \multirow{2}{*}{\multicolumn{2}{|c|}{$\begin{array}{r}48,94 \\
\pm 0,68\end{array}$}} & \multirow{2}{*}{\multicolumn{2}{|c|}{$\begin{array}{r}5^{2,36} \\
\pm 0,90\end{array}$}} & \multirow{2}{*}{\multicolumn{2}{|c|}{$\begin{array}{r}51,56 \\
\pm 1,13\end{array}$}} & \multirow{2}{*}{\multicolumn{2}{|c|}{$\begin{array}{r}51, \text { I } 5 \\
\pm 0,64\end{array}$}} \\
\hline E. S. & & & & & & & & \\
\hline
\end{tabular}


土I,I3) e con quelli calcolati sui dati di Lynch (I936), Allan e coll. (I948) e Golub (I953), rispettivamente 49,$73 ; 53$, I9; 50, I5.

Di particolare dalla nostra casistica appare una differenza fra le età medie di manifestazione dei carcinomi solidi primitivi e dei cistocarcinomi. Per i primi sarebbe

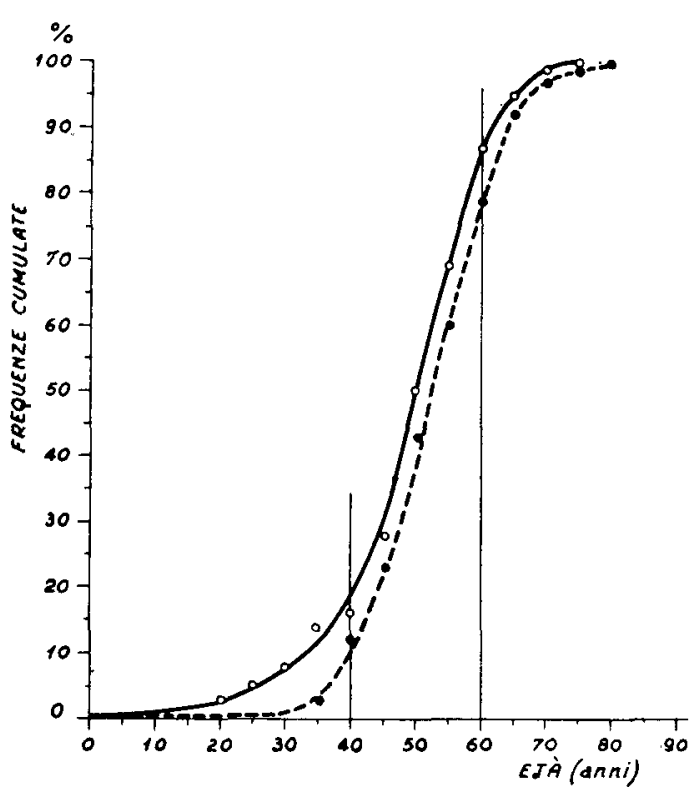

Fig. I - Gurve di graduazione relative alle pazienti affette da carcinoma solido primitivo (-) e da cistocarcinoma $(\ldots \ldots \ldots \ldots)$ $48,94 \pm 0,68$ anni, per gli altri $52,36 \pm 0,90$ anni: differenza che è da ritenere significativa, essendo $\mathrm{t}=3,026$ e quindi $\mathrm{P}$ $<$ o,or. Sembra dunque che i cistocarcinomi tendano a manifestarsi in età più avanzata dei carcinomi solidi primitivi. $\mathrm{E}$ ciò è messo meglio in evidenza dalle frequenze percentuali cumulate $(\Sigma \mathrm{f} \%)$ indicate nella stessa tabella 4 e dalle rispettive curve di graduazione (fig. I), dalle quali si rileva come, pur essendo approssimativamente uguali le frequenze dei casi fra 40-6o anni per i due tipi, 70,20\% per i carcinomi solidi e $7 \mathrm{I}, 30 \%$ per i cistocarcinomi, fra 6o-8o anni la frequenza dei cistocarcinomi è notevolmente superiore $(2 \mathrm{I}, 30 \%)$ a quella dei carcinomi solidi ( $13,62 \%)$, mentre questi sono più frequenti $(\mathrm{I} 6,22 \%)$ dei cistocarcinomi $(7,4 \mathrm{I} \%)$ fino a 40 anni.

E parso di un certo interesse analizzare pure come sono variamente colpite le diverse classi di età. Nella figura 2 sono tracciati il grafico delle frequenze relative $\left(f_{c}\right)$ delle donne affette da cancro ovarico distribuite per classi di cinque anni, come risulta dal nostro campione, supposto rappresentativo della rispettiva popolazione; ed il grafico delle frequenze relative $\left(f_{p}\right)$ dei soggetti della popolazione dell'Italia del Nord appartenenti alle stesse classi di anni, in base al censimento del I95 1 , anno che si trova a metà tra il 1946 ed il I 955 ai quali si estende il nostro materiale. Si è scelta la popolazione generale invece della femminile mancando per quest'ultima nelle statistiche ufficiali la distribuzione per quinquenni al di sopra dei 60 anni e non essendoci d'altra parte una sensibile differenza tra le due serie di frequenze.

Dal confronto dell'andamento delle due curve è facile rilevare che l'incidenza della malattia nei diversi gruppi di età varia considerevolmente. Ma per precisare meglio il fenomeno, calcolati per ogni quinquennio i rapporti tra le due frequenze $\left(f_{c} / f_{b}\right)$, si sono riportati in diagramma (fig. 3) i valori trovati, ottenendo cosi una rappresentazione della variazione dell'incidenza delle cancerose col variare dell'età. $\mathrm{Si}$ vede in tal modo - pur non potendo per questa via determinare il rischio effettivo 
di morbilità per le diverse età - come ad una scarsa incidenza dei carcinomi ovarici fino ai 30 anni che cresce leggermente fra 30 e 40 anni, segue un brusco aumento da 40 a $5^{\circ}$ anni con un massimo fra $45^{-5}$ e poi una lenta diminuzione fino a $6_{5}$ anni, oltrepassati i quali si ha quasi la scomparsa improvvisa.

Per i carcinomi ovarici non vale dunque quanto è stato registrato per altre neoplasie quali, secondo l'affermazione di Marks e col. (1948), i cancri dello stomaco, della mammella, dell'utero, della vescica, della pelle e della cavità boccale, nei quali l'incidenza aumenta costantemente con l'età.

Ripromettendoci di ritornare su questo argomento in altra sede per il valore che può assumere la variazione della frequenza di una data manifestazione morbosa con l'età in un'interpretazione eziologico-ereditaria della stessa, intendiamo per ora sottolineare soltanto il fatto che la massima frequenza d'incidenza fra i 40-6o anni caratterizza i carcinomi rispetto agli altri oncotipi ovarici: i disgerminomi,

Fig. 2 - Distribuzione delle frequenze relative per età dei soggetti appartenenti alla popolazione generale (..........) e al campione delle pazienti affette da cancro ovarico

Fig. 3 - Variazione dell'inFig. 2
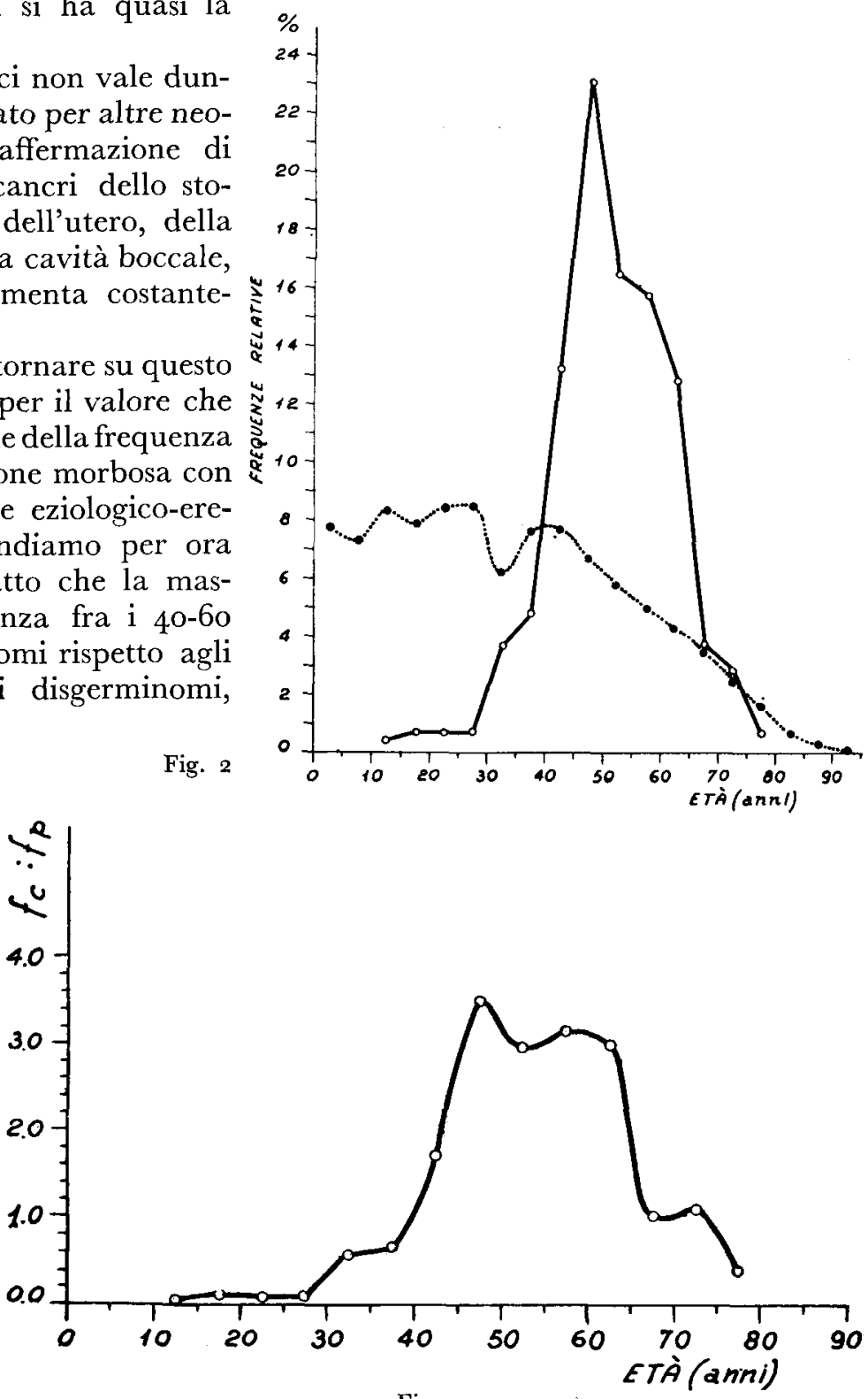
rico con l'età.

Fig. 3 
frequenti in età prepubere, si manifestano in media sui 20 anni; gli arrenoblastomi tra i 20-40 anni, i teratomi in età giovanile, i tumori delle cellule a granulosa in tutte le età. Per tal motivo sarebbe, a nostro avviso, di una certa utilità ai fini di un'indagine eziopatogenetica, avendo a disposizione una casistica più ampia, studiare pure se esiste una correlazione fra età di manifestazione della malattia ed età di menopausa.

\section{Fertilità delle pazienti affette da cancro ovarico}

Un secondo elemento di notevole importanza clinica e soprattutto genetica è la fertilità delle pazienti affette da carcinomi ovarici.

Escluse le 52 pazienti nubili, che costituiscono il $19,63 \%$ dei 265 casi per cui è stato accertato lo stato civile, si è preso in considerazione il numero dei concepimenti, dei nati (vivi e morti) e degli aborti delle pazienti sposate per le quali il dato risultava con sicurezza.

Dalla tabella 5 si ricava che il $16,43 \%$ (35 su 2 I3) di esse sono nulligravide, pur avendo 27 su 35 età superiore ai 40 anni al momento del rilevamento del dato. Tenendo conto anche del 5 , I I \% ( I su 2 I 3 ) delle pazienti con soli aborti si raggiunge il 2 I, $54 \%$ di nullipare.

Tabella 5 - Parità nelle pazienti affette da cancro ovarico

\begin{tabular}{|c|c|c|c|}
\hline \multirow{2}{*}{$\begin{array}{l}\text { Numero di } \\
\text { concepimenti }\end{array}$} & \multicolumn{3}{|c|}{ Numero di } \\
\hline & pazienti & nati & aborti \\
\hline o & 35 & 一 & 一 \\
\hline $\mathbf{I}$ & $5^{I}$ & 44 & 7 \\
\hline 2 & $5^{I}$ & 84 & I 8 \\
\hline 3 & $3^{2}$ & 88 & 8 \\
\hline 4 & I 8 & 62 & ro \\
\hline 5 & I 4 & 55 & 15 \\
\hline 6 & 5 & 28 & 2 \\
\hline 7 & 2 & I3 & I \\
\hline 8 & I & 6 & 2 \\
\hline 9 & $\mathbf{I}$ & 7 & 2 \\
\hline Io & $\ldots .$. & - & - \\
\hline I I & 2 & I9 & 3 \\
\hline 12 & - & 一 & - \\
\hline I3 & $\mathbf{I}$ & 12 & I \\
\hline Totali & 213 & $4^{18}$ & 69 \\
\hline $\begin{array}{l}\text { Medie } \\
\text { E. S. }\end{array}$ & & $\begin{array}{r}1,96 \\
\pm 0,13\end{array}$ & $\begin{array}{r}0,34 \\
\pm 0,05\end{array}$ \\
\hline
\end{tabular}


Questa frequenza di nullipare, superiore all' I I,4\% di Montgomery (I948), è tuttavia inferiore al 3I\% del Lynch (I936), al 40\% del Dockerty (I945), al $32 \%$ del Munnel (1952), al 31,6\% del Golub (1953). Gli AA. citati sono concordi nell'ammettere che la sterilità fra le carcinomatose ovariche - considerando come sterili le nulligravide e le nullipare con aborti - è nettamente superiore al tasso di sterilità calcolato dalle statistiche ufficiali (Munnel, r952) o su gruppi di altre pazienti ginecologiche non carcinomatose (Golub, 1953). Si è cercata una conferma per altra via: confrontando cioè il valore della fecondità stimato per la popolazione italiana con quello stimato sul nostro campione di carcinomatose ovariche.

Nella tabella 6 è riportato, per gli anni che ci interessano, il numero medio dei figli per matrimonio, secondo il computo eseguito, in base alle statistiche ufficiali, dal Lenti (1950) - che considerò come figli tanto i nati vivi che i nati morti e da Henry e Pressat (I955) - che considerarono come figli soltanto i nati vivi.

\begin{tabular}{c|c|c} 
Tabella 6 - Fecondità della popolazione italiana \\
\hline \hline \multirow{2}{*}{ Anno } & N. medio di figli per matrimonio \\
\hline & Lenti & Henry-Pressat \\
\hline & & \\
1945 & 2,89 & 2,80 \\
1946 & 3,53 & 3,44 \\
1947 & 3,23 & 3,15 \\
$194^{8}$ & 3,09 & 2,99 \\
1949 & 2,85 & 2,76 \\
$195^{\circ}$ & 2,74 & 2,66 \\
$195^{1}$ & & 2,50 \\
$195^{2}$ & & 2,46 \\
1953 & & 2,43 \\
1954 & & 2,44
\end{tabular}

Riferendoci a questa tabella, si constata che il numero medio dei figli (nati vivi+ nati morti) per matrimonio nel nostro campione, costituito dalle pazienti coniugate cancerose appartenenti al periodo I946- I955, risultante di $1,96 \pm 0$, I 3 , con limiti fiduciali al $95 \%$ di probabilità di $\mathrm{I}, 70-2,22$, è significativamente inferiore a quello della popolazione generale secondo i dati sia del Lenti che di Henry e Pressat.

Si deve perciò ammettere che con il cancro ovarico sia connessa una diminuzione della fertilità: fatto non osservato invece presso le pazienti di cancro dell'utero, per le quali il numero medio dei figli si accorda bene, secondo i dati di Beolchini e coll. (1956), con quello della popolazione generale.

La diminuzione della fertilità non appare tuttavia da ascrivere ad una aumentata abortività. Questa ammonta infatti al 13,7\% sul totale dei concepimenti. Frequenza non lontana da quella che si attenderebbe nella popolazione milanese se si accettano le stime, trovata (17,07\%) e corretta (20-25\%), di Alberti (1932) e Boldrini (I946) riferentisi ad una popolazione di $5.74^{8}$ donne ed un totale di 2 I.o92 parti e se si tien 
conto che questi AA. considerano nelle loro ricerche, per uniformità di criterio, come aborti «tutti i partoriti morti a tutte le epoche di gestazione ». Del resto la nostra media di aborti per donna $(0,34 \pm 0,05)$ è leggermente inferiore a quelle trovate da Beolchini e coll. ( I956) per le pazienti di cancro dell'utero $(0,4 \pm 0, \mathrm{I}$ per il corpo e $0,6 \pm 0,02$ per il collo) che non mostrano, come è stato detto, diminuzione di fertilità.

\section{Discussione}

Due dati sono stati particolarmente messi in rilievo dall'analisi dei 275 casi di carcinoma ovarico presi in considerazione:

I. Massima frequenza dei carcinomi ovarici (carcinomi solidi primitivi e cistocarcinomi) fra i 40-6o anni, periodo in cui solitamente si manifesta il climaterio.

2. Minor fertilità delle donne affette da cancro ovarico rispetto alla popolazione generale.

Sebbene un tentativo di dare un'interpretazione unitaria di questo comportamento, che sembra peculiare dei carcinomi ovarici, esorbiti un poco dai limiti del nostro lavoro, potendo tuttavia interessare anche il campo genetico ed essendocene offerta l'occasione dai rilievi fatti, ci siamo prospettati il quesito se gli squilibri ormonali ipofiso-ovarici (ipofollicolinismo e iperproduzione di ormoni gonadotropi), caratteristici sia del climaterio che di certe forme di sterilità, possano esserne invocati quale causa o almeno quale fattore principale.

Tale quesito è suggerito dall'ipotesi dell'eziopatogenesi ormonale di alcuni tumori ovarici, avanzata da qualche studioso in seguito a ricerche sperimentali. Ricordiamo, tra le altre, quelle dei Biskind (1944), di Li e Gardner (I947-1949) e di Muhlbock (I95I) i quali riuscirono a stimolare la formazione di tumori ovarici (tumori a cellule della granulosa e luteomi) provocando artificialmente degli squilibri ipofiso-ovarici nel senso di una protratta sovrapproduzione di ormoni gonadotropi accompagnata da deficienza o assenza di estrogeni. Ipotesi che è stata generalizzata da Muhlbock (I95I) scrivendo al termine del suo lavoro dopo aver brevemente discusso se quanto era stato trovato sperimentalmente poteva estendersi ai tumori ovarici della donna: «Le osservazioni fatte sinora danno diritto all'ipotesi che i tumori dell'ovaio sono causati da un disordine nell'equilibrio ormonale » (pag. 3678).

Per una certa analogia si potrebbe, è vero, opinare che anche i carcinomi ovarici tendano ad insorgere come una risposta agli squilibri ormonali - simili a quelli sperimentalmente provocati - che si verificano soprattutto nel periodo del climaterio ed in alcune forme di sterilità: in questi casi, agendo tale fattore su follicoli o su tessuti in cui è notevolmente diminuita la facoltà di maturare, si avrebbe lo sviluppo non di tumori funzionanti, ma di tumori più altamente maligni. La condizione essenziale che qui pure siano interessati il tessuto follicolare o germinativo si può ritenere come assicurata dagli studi sull'origine istogenetica dei carcinomi solidi primitivi e dei cistocarcinomi sierosi, ricondotta da un gran numero di Autori all'epitelio germinativo o follicolare. 
Però mentre da un lato pare da accettare l'importanza dell'indicato squilibrio ormonale quale fattore generico di stimolazione tumorale, non sembra dall'altro che esso possa considerarsi quale causa o fattore principale dell'insorgenza dei carcinomi: prima di tutto perchè sperimentalmente si sono finora ottenuti solo tumori funzionanti e poi per la relativa rarità delle manifestazioni neoplastiche da noi considerate. $\grave{\mathrm{E}}$ in particolare questa relativa rarità che farebbe postulare l'esistenza di un fattore idiotipico a cui spetterebbe la funzione principale di realizzare lo sviluppo della formazione cancerosa, la quale si estrinsecherebbe probabilmente soltanto in concomitanza del fattore "squilibrio ormonale » del tipo ricordato.

Questa, ad ogni modo, non è che un'ipotesi di lavoro alla cui convalida potranno forse portare un contributo anche le ricerche iniziate sotto l'aspetto genetico.

\section{Conclusioni}

I rilievi più importanti per la nostra ricerca genetica, occorsi nell'esame di 275 pazienti affette da carcinoma ovarico, le quali rappresentano $\mathrm{i}$ «casi primari» per l'indagine genealogica sulla familiarità del cancro ovarico, si riconducono ai seguenti:

I. Il periodo in cui con la maggiore probabilità una donna può essere colpita da carcinoma ovarico si estende dai 40 ai 60 anni con un massimo fra $45^{-50}$. Tra $i$ Io-40 anni è più probabile che sia colpita da carcinoma solido; tra 6o-8o da cistocarcinoma.

2. Nonostante che la massima frequenza di morbilità coincida con il periodo climaterico, la funzione riproduttiva delle pazienti è complessivamente alterata, risultando in una diminuzione della fertilità media.

3. Alla possibilità di interpretare questi dati come una conferma dell'ipotesi ormonale della carcinogenesi ovarica sembra da preferire una spiegazione eredopatogenetica, che esige tuttavia - per essere stabilmente fondata - ulteriori ricerche in varie direzioni, tra cui quella genetica. 


\section{Riassunto}

Gli Autori, avendo iniziato una ricerca sulla familiarità del cancro ovarico, in questa prima parte del lavoro:

I. Definiscono il gruppo dei «casi primari », che risulta costituito da 74 casi di carcinoma solido primitivo, 108 casi di cistocarcinoma e 93 casi di tumore maligno dell'ovaio non ulteriormente classificati.

2. Esaminano l'età di manifestazione dei carcinomi ovarici e la variazione dell'incidenza della malattia con l'età, trovando.

a) quale età media di manifestazione $5 \mathrm{I}, \mathrm{I} 5 \pm 0,64$ anni, che però scende a $48,94 \pm 0,68$ anni per $i$ carcinomi solidi primitivi e sale a $5^{2,36} \pm 0,90$ anni per $i$ cistocarcinomi;

b) quale periodo della massima incidenza quello fra $40-60$ anni, seguito da una netta e brusca diminuzione oltre i 65 anni.

3. Analizzano la fertilità delle pazienti affette da cancro ovarico, registrando una diminuzione della fertilità media del $29 \%$ rispetto alla popolazione generale.

4. Accennano all'importanza del fattore ormonale quale condizione allo sviluppo del cancro ovarico, che sembra tuttavia esigere altri fattori di ordine probabilmente idiotipico.

\section{Bibliografia}

I. Abruzzese G.: La predisposizione costituzionale ai tumori negli organi genitali nella donna. Rivista italiana di Ginecologia, I 7, 1-I I 5, 1934 .

2. Alberti S.: Sulla frequenza degli aborti nella popolazione milanese desunta da osservazioni ospitaliere. Congresso Italiano per gli studi sulla popolazione, Roma 1932, estratto.

3. Allan M. S. e Hertig A. T.: Carcinoma of the ovary. Am.J.Obst. and Gynec., 58, 640-653, 1949.

4. Barzilai G.: Atlante dei tumori dell'ovaio, Parma, 1949.

5. Beolchini P.E., Cresseri A., Gianferrari L., Malcovati P. e Morganti G.: Ricerche genetiche sulle neoplasie dell'utero. A.GE.ME.GE. 5, 462-468, 1956.

6. Biskind M. S. e Biskind G.R.: cit. in Sirtori C.

7. Boldrini M.: Demografia, Milano, 1946.

8. Dockerty M. B.: Ovarian neoplasms. A collective review of the recent literature. Surg. Gynec. and Obst., 8I, I 79-204, I 945 .

9. Ferraris F. e Bongiovanni G.: La classificazione dei tumori ovarici. Ann. Ost. Ginec., 77, 705-723, i955.

10. Gedda L.: Studio dei Gemelli, Roma, 195 I.

I1. Golub L. J.: The diagnosis of ovarian cancer. Am. J. Obst. and Gynec. 66, 169-177, 1953.

12. Henry L. e Pressat R.: Evolution de la fécondité en Italie. Population, io, 501-528, i955.

13. Lentr L.: La fecondità matrimoniale in Italia dal r930 al r950. Giorn. degli Economisti e Annali di Economia, 12, 527-572, 1953.

14. Li M. H. e Gardner W. U.: cit. in Sirtori C.

15. Liber A. F.: Cancer of the ovary in a mother and five daughters. Arch. of Pathol., 49, 280-295, 1950.

I6. LxNch F. W.: A clinical review of 1 lo cases of ovarian carcinoma. Am. J. Obst. and Gynec., 32, 759-777, 1936 .

17. Malcovati P.: Le moderne vedute sull'istopatogenesi e classificazione dei tumori ovarici. Clin. Ost. Ginec., I 948 , estratto. 
Soini A., Serra A.: Ricerche sulla familiarità del carcinoma ovarico

18. Marks J. H. e Wittenborg M. H.: Results of treatment of carcinoma of the ovary with data on the age incidence of this disease. Surg. Gynec. and Obst., 87, 541-545, 1948.

rg. Montgomery J. B.: Malignant tumors of the ovary. Am. J. Obst. and Gynec., 55, $201-215,1948$.

20. Murlbock O.: Onderzoekingen over de genese der ovariumtumoren. Nederlandsch Tijdschrift voor Geneeskunde, 95, $3^{6} 72-3^{679}$, I95I.

2 I. Munnel E. W.: Ovarian Carcinoma. Predisposing factors, diagnosis, treatment. Cancer, 5, I I $28-1$ I 33 , I 952 .

22. Nìgre E.: Tumeurs malignes de l'ovaire. Presse Médical, 59, 708-C, 1948.

23. Novak E.: Gynecologic and Obstetric Pathology. Philadelphia, 1952.

24. RAO C. R.: Advanced statistical methods in biometric research, New York, I952.

25. Scaglione S. : Glassificazione dei tumori Ovarici. Atti Soc. Ital. Ost. Ginec. 49, 1-29, 1948

26. Schiller: Concepts of a new classification of ovarian tumors. Surg. Gynec. and Obst., 70, 773-782, 1940.

27. Schmid H. H.: Eierstockkrebs. Der Krebsarzt, 9, 344-352, $195^{2}$.

28. Selye H.: cit in Liber A. F.

29. Sirtorı C.: La terapia medica dei tumori. Milano, I953.

30. Von Verschuer O. e Kober E.: Die Frage der erblichen Disposition zum Krebs. Ergebnis einer Forschung durch 20 Jahre an einer auslesefreien Zwillingsserie, Wiesbaden, 1956.

\section{RÉSUMÉ}

Les Auteurs ont commencé une recherche génétique sur le cancer ovarien. Dans cette première partie du travail ils ont:

1. déterminé le groupe des "cas primaires", constitué de 74 cas de carcinome solide, de 108 cas de carcinome cystique et de 93 cas de tumeur maligne de l'ovaire non autrement classifiés.

2. étudié l'âge de manifestation des carcinomes ovariens et la variation de la fréquence de la maladie avec l'âge, de quoi on voit que:

a) l'âge moyenne de manifestation est de $51,1 \varsigma \pm 0,64$ ans pour le cancer en général, mais de 48,94 $\pm 0,68$ ans pour le carcinomes solides et de $52,36 \pm 0,90$ ans pour les carcinomes cystiques;

b) l'âge de la fréquence plus élevée se place entre 40-60 ans, suivie d'une nette diminution après 65 ans;

3. analysé la fécondité des patients, et notent une diminution de la fécondité moyenne du $29 \%$ relativement a celle de la population générale;

4. touché l'importance d'un facteur hormonal conditionnant le développement du cancer ovarien, qui semble néammoins exiger d'autres facteurs probablement idiotypiques. 


\section{SUMMARY}

The Autors have begun a genetic research on the ovarian cancer. In this first part of the work:

1. it has been defined the group of the "primary cases" in which have been included 74 cases of solid carcinoma, 108 cases of cystic carcinoma and 93 cases of unclassified malignant tumor of the ovary.

2. it has been studied the age of appearance and the age incidence variation of the ovarian cancer, and it has been found that:

a) the mean age of appearance is 51,15 $\pm 0,64$ years, but it lowers to $48,94 \pm 0,68$ years for the solid carcinoma and rises to 52,36 $\pm 0,90$ years for the cystic carcinoma;

b) the period of the greatest frequency of the disease is between 40-60 years of age, followed by a sharp decrease over age 65 ;

3 . it has been analysed the fertility of the patients, and it has ben recorded a decrease of the mean fertility up to $29 \%$ as compared to the general population;

4. it has been mentioned the importance of the hormonal factor as a problable condition for the development of the ovarian cancer, that nevertheless calls for other - likely idiotypic - factors.

\section{ZUSAMMENFASSUNG}

Die Verfasser haben eine Familienforschung über Eierstockkrebs eingeleitet. In diesem ersten Teil der Arbeit:

1. bestimmen sie die "Ausgangsfälle » wie folgt: 74 Fälle von primären massiven Karzinomen, 108 Fälle von Kystokarzinomen und 93 Fälle von bösartigen nicht klassifizierten 'Tumoren;

2. untersuchen sie das Alter, in dem der Eierstockkrebs in Erscheinung tritt, und die Veränderung der Frequenz der Krankheit nach dem Alter, und finden:

a) als Durchschnittsalter $51,15 \pm 0,64$ Jahre, die jedoch bei den primären massiven Karzinomen bis $\mathrm{zu} 48,94 \pm 0,68$ Jahre herabsinken und bei den Kystokarzinomen bis zu $52,36 \pm 0,90$ steigen;

b) als Zeitabschnitt der grossten Häufigkeit die Jahre 40-60, dem nach den fünfundsechziger Jahren eine eindeutige und entschiedene Verringerung folgt;

3. dann untersuchen sie die Fruchtbarkeit der an Eierstockkrebs Erkrankten und stellen eine Verminderung von $29 \%$ der Durchschnittsfruchtbarkeit im Vergleich zur Gesammtbevölkerung fest;

4. und weisen endlich hin auf die Bedeutung des Hormomen-Faktors als Bedingung des Fintretens des Eierstockkrebs, das jedoch auch andere Faktoren, wahrscheinlich idiotypischer Art, $\mathrm{zu}$ fordern scheint. 